\title{
Regenerative synchronous electrical charge extraction for highly coupled piezoelectric generators
}

\author{
Adrien Morel, Gaël Pillonnet \\ Univ. Grenoble Alpes, F-38000 Grenoble, France \\ CEA, LETI, MINATEC, F-38054 Grenoble, France \\ adrien.morel@cea.fr
}

\begin{abstract}
This paper proposes a new strategy for vibration harvesting using piezoelectric material. This work relies on an adaptation of the classical Synchronous Electrical Charge Extraction (SECE). Instead of harvesting the energy at every displacement extremum, we choose to wait a certain number of extremum before harvesting the accumulated energy. This technique extends the harvested power compared to SECE, especially with highly coupled piezoelectric generators. It is notably shown that this strategy largely increases the harvested power as the product $k_{m}{ }^{2} Q_{m}$ gets greater, and at the same time optimizes the switching losses. Measurements on a piezoelectric energy harvester (PEH) exhibiting a relatively large global coupling coefficient have been realized and have proven the efficiency and potential of this technique $(193 \%$ harvested power improvement at resonance compared with a standard SECE strategy).
\end{abstract}

Keywords-Vibrations Harvesting, Multiphysics modeling, Piezoelectricity, Interface circuit, Extraction strategy, SECE.

\section{INTRODUCTION}

Recently, there has seen a growing interest in new energy harvesting sources for powering sensors nodes. Piezoelectric elements are of particular interest for applications in closed confined environment, because of their high energy densities and integration potential.

Interface circuits for Piezoelectric Generators (PEG) are a key point in the energy conversion chain. For lowly coupled piezoelectric harvesters, it has been demonstrated that synchronous strategies like Synchronous Electrical Charge Extraction (SECE) are promising and exhibit higher performances than classic electronic interfaces, such as a diode rectifier followed by a buck-boost converter [1-3]. However, for highly coupled or lowly damped generators, SECE is no longer efficient since it overdamps the mechanical system. For this very particular kind of harvester, researchers have started to find new strategies in order to optimize the damping induced by the electrical interface and even tune the resonant frequency of the whole electromechanical system, based on the electrical interface [4-6].

In this paper, we propose to use a $N-S E C E$, a new strategy for high coupling or low damping generators. We first introduce the unified electromechanical model used to predict the effects of the electrical interface on the overall system. We develop the analytical equations leading to the harvested power using the $N$ $S E C E$. Then, we present the experimental validation realized on

\author{
Adrien Badel \\ Univ. Savoie Mont Blanc, SYMME, \\ F-74000 Annecy, France
}

a highly coupled piezoelectric generator, and finally propose an ASIC implementation of the proposed strategy.

\section{MOdeling OF THE N-SECE}

\section{A. Piezoelectric energy harvester modeling}

In order to express the equations governing the dynamic of the piezoelectric system, we use the general William and Yates electromechanical model. Considering the generic mechanical to electrical model shown by Fig. 1, and described in [1], the electromechanical constitutive equations can be written as:

$$
\left\{\begin{array}{c}
M \cdot \ddot{x}+D \cdot \dot{x}+K_{s c} \cdot x+\alpha \cdot v_{p}=\mathrm{F}=M \cdot \ddot{y} \\
i=\alpha \cdot \dot{x}-C_{p} \cdot v_{p}-\frac{v_{p}}{R_{p}} \\
x(t)=X_{m} \cos (\theta)=X_{m} \cos (\omega t)
\end{array}\right.
$$

where $y, x, F, v_{p}$ and $i$ respectively stand for the ambient displacement, the dynamic mass displacement, the vibration force applied on the whole system, the piezoelectric output voltage and current. $M$ is the dynamic mass of the system, $K_{S C}$ is the short-circuited stiffness, $D$ is the mechanical damping of the structure, $C_{p}$ is the piezoelectric capacitance, $R_{p}$ represents the dielectric losses in the piezoelectric material, and $\alpha$ is the electromechanical coupling between the mechanical part and the electrical part of the piezoelectric generator. We will consider in this paper that the displacement is purely sinusoidal and with a constant amplitude $X_{m}$, in order to simplify the theoretical developments, as shown in (1).

These equations (1) are widely used [4,6] as they model very accurately the electromechanical behavior of any piezoelectric harvester, as long as the mechanical part of the system is linear and doesn't exhibit strong resonance modes.

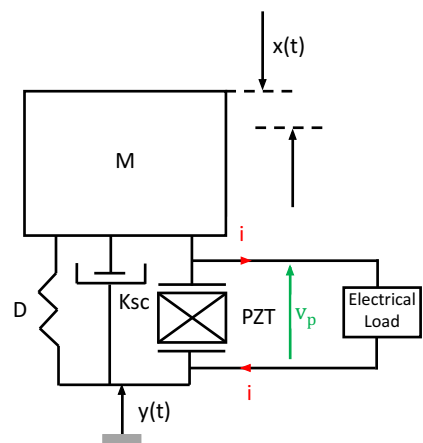

Fig. 1. Electromechanical model. 


\section{B. Expression of the piezoelectric voltage}

In order to solve (1), we need to first find the expression of the piezoelectric voltage $v_{p}$. To do so, we will split up its expression on a time period. We will consider that the dielectric losses $R_{p}$ are negligible and have no impact on the harvested power, as it will greatly simplify the theoretical developments. The variable $n$ stands for the number of extremum of displacement between two harvesting events.

A simple system realizing this operation can be imagined as shown on Fig. 2. Every $n$ voltage (or displacement) extremum, an inductance is connected to the piezoelectric generator. The energy stored in the piezoelectric capacitance is then transferred in the inductance as the piezoelectric voltage becomes null. When the voltage is equal to 0 , the inductance is disconnected and the energy is finally transferred from the inductance into a storage circuit.

The piezoelectric voltage waveforms have been simulated numerically using a Simulink block diagram in Matlab for different $n$, and can be observed on Fig. 3 . We can notice that as $n$ gets greater, the harvested voltage increases. The analytic expression of the piezoelectric voltage is given by the system of equations (2).

$$
\mathrm{v}_{\mathrm{p}_{\mathrm{n}}}(\theta)=\left\{\begin{array}{rr}
\left.\frac{1}{\mathrm{C}_{\mathrm{p}}} \int_{0}^{\theta} \alpha \cdot \dot{x}_{n}(\theta) \mathrm{d} \theta, \quad \forall \theta \in\right] 0, \pi[ \\
0, \text { if } \theta=\pi \\
\left.\frac{1}{\mathrm{C}_{\mathrm{p}} \int_{\pi}^{\theta} \alpha \cdot \dot{x}_{n}(\theta) \mathrm{d} \theta, \quad} \quad \forall \theta \in\right] \pi, 2 \pi[ \\
0, \text { if } \theta=2 \pi
\end{array}\right.
$$

As shown by (2), $v_{p_{n}}$ is non-linear. In order to inject $v_{p_{n}}$ in (1), we need to linearize it. Using Fourier series, and considering that only the $n^{\text {th }}$ harmonic has an impact on the system dynamic due to filtering effect of the mechanical resonator, we find the expression of the $n^{\text {th }}$ harmonic of the piezoelectric voltage, given by (3).

$$
\left\{\begin{array}{c}
\mathrm{v}_{\mathrm{p}_{\mathrm{n}}}(\theta)=\frac{\alpha}{C_{p}}\left[1-i \cdot 2 \frac{1}{\pi \cdot n} \cdot\left((-1)^{\mathrm{n}}-1\right)\right] \cdot \underline{x_{n}}(\theta) \\
\underline{x_{n}}(\theta)=X_{m_{n}} \cdot e^{i \theta}
\end{array}\right.
$$

Where $\underline{x}_{n}$ is the displacement written in the Fourier domain.

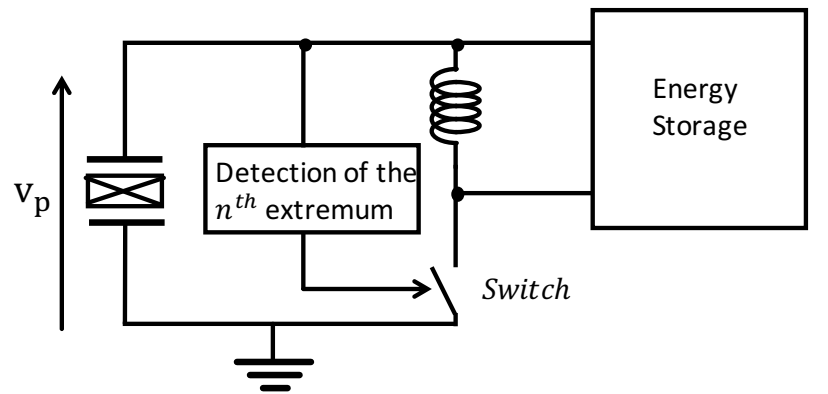

Fig. 2. N-SECE based on a $n^{\text {th }}$ extremum detection.

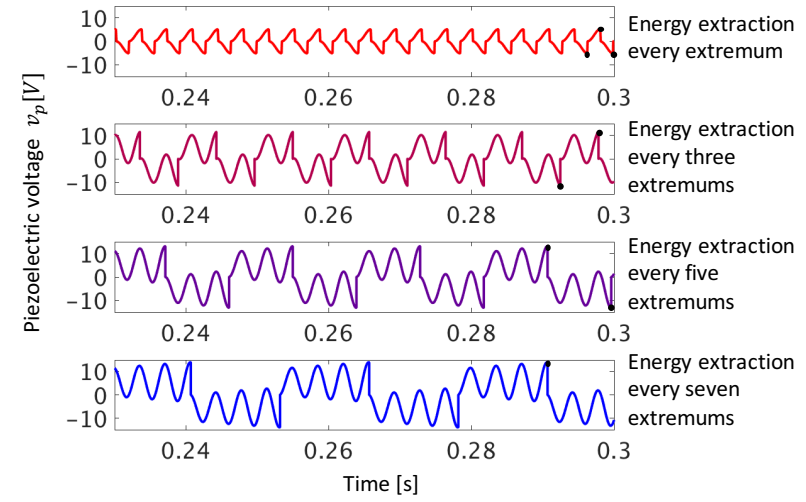

Fig. 3. Simulated waveforms of the piezoelectric voltage with $n=1,3,5,7$.

\section{Expression of the harvested power}

We can now inject (3) in (1) in order to find the displacement amplitude. Solving the differential equation by applying the Laplace transform, we eventually find the displacement amplitude, given by (4).

$$
\left\{\begin{array}{c}
X_{m_{n}}=\frac{F}{\sqrt{A^{2}+B^{2}}} \\
A=K_{s c}-M \omega^{2}+\frac{\alpha^{2}}{C_{p}} \\
B=\omega D-2 \frac{\alpha^{2}}{\pi C_{p} n}\left((-1)^{\mathrm{n}}-1\right)
\end{array}\right.
$$

We can see that the variable $n$ has only an influence on the damping of the system, but doesn't modify the electromechanical resonant frequency. The greater $n$ is, the less the system will be damped. The harvested energy is the energy stored in the piezoelectric material right before the harvesting process, which happens every $n / 2$ period. The harvested power can be expressed as a function of the displacement amplitude, as shown by (5).

$$
\mathrm{P}_{\mathrm{n}}=\frac{\omega \cdot \alpha^{2}}{2 \pi \cdot n \cdot C_{p}} X_{m_{n}^{2}}^{2}\left((-1)^{\mathrm{n}}-1\right)^{2}
$$

\section{THEORETICAL ANALYSIS OF THE PROPOSED STRATEGY}

\section{A. Performances at resonance with different coupling}

As expressed by (5), if $n$ is even, the harvested power is null. However, if $n$ is an odd number, the harvested power is not null and can hence be analyzed. When $n=1$, the energy stored in the dielectric capacitance of the piezoelectric material is harvested at every displacement extremum. This is the socalled SECE (Synchronous Electrical Charge Extraction) strategy. Interestingly enough, when $n$ increases (implying fewer harvesting events), the power doesn't necessarily decrease, as shown on Fig. 4 and Fig. 5. 


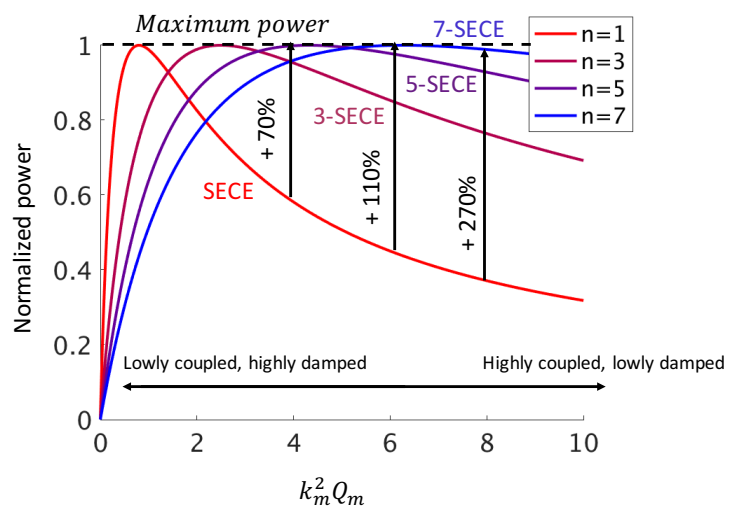

Fig. 4. Harvested power at open circuit resonance as a function of $k_{m}^{2} Q_{m}$.

Here, the normalized power is defined as $P_{n} / P_{\max }$, with $P_{n}$ the net harvested power for a given $n$ and $P_{\text {max }}$ the maximum harvestable power at a fixed acceleration and mechanical damping, given by $P_{\max }=\frac{F^{2}}{8 D} \cdot k_{m}^{2}$ is the squared normalized electromechanical coupling and $Q_{m}$ the mechanical quality factor of the piezoelectric generator, as defined in [6]. If the figure of merit $k_{m}^{2} Q_{m}$ is greater than 2 , harvesting the energy every demi-period becomes less interesting because the system is too highly coupled. Indeed, the damping induced by the harvesting processes is getting too important, leading to a reduction of the seismic mass displacement magnitude and consequently of the harvested power. We can observe that the greater the coupling, the greater $n$ should be in order to optimize the harvesting process. For example, as shown on Fig. 4, a generator having its $k_{m}^{2} Q_{m}$ equals to 4 should be used with an electrical interface configured with $n=5$ in order to maximize the extracted energy.

\section{B. Performances around the open circuit resonance frequency}

Fig. 5 shows how the harvested power spectrum can be theoretically increased on a highly coupled PEG by adjusting the number of harvesting events. The closer the generator works from its open circuit resonant frequency, the higher $n$ should be in order to minimize the damping processes. However, lower $n$ values become optimal as the frequency shifts away from the open circuit resonance frequency, since the damping should be increased in order to enlarge the harvesting spectrum. The damping induced by the electrical interface on the piezoelectric energy harvester is hence inversely proportional to $n$ and is given by (6).

$$
\mathrm{D}_{\mathrm{e}_{\mathrm{n}}}=-\frac{1}{n} \cdot \frac{2 \cdot \alpha^{2}}{\pi \cdot C_{p} \cdot \omega}\left((-1)^{\mathrm{n}}-1\right)
$$

The normalized frequency is given by $\omega / \omega_{0}$, where $\omega_{0}$ is the short-circuited mechanical resonant frequency of the harvester. The power curves in Fig. 5 are centered around a normalized frequency corresponding to the open circuit resonant frequency of the harvester, which is different from the mechanical short-circuit resonant frequency, because of the high backward electromechanical coupling.

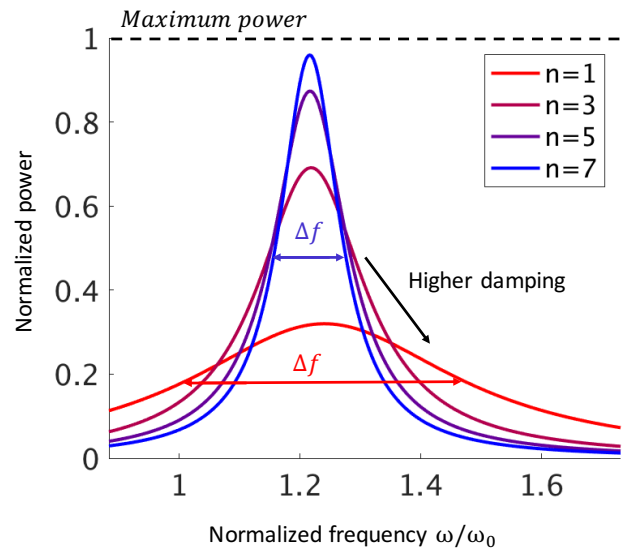

Fig. 5. Harvested power spectrum with $k_{m}^{2} Q_{m}=10$.

\section{EXPERIMENTAL VALIDATION}

\section{A. Piezoelectric generator characteristics}

In order to validate the proposed electrical interface, a highly coupled piezoelectric generator composed of single crystal PZN-PT plates deposited on a cantilever beam has been used. The characteristics of the piezoelectric generator (Fig. 6) are shown in Table 1, and have been determined empirically, thanks to an impedance analyzer. This generator has been excited using an electromagnetic shaker, and its displacement, acceleration and voltage have been observed.

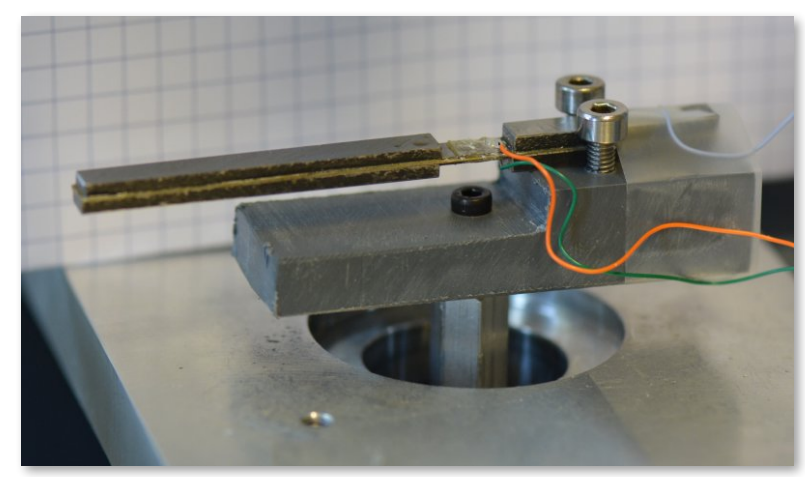

Fig. 6. Highly coupled piezoelectric generator with $10 \times 5 \times 0.5 \mathrm{~mm}^{3}$ plates of PZN-PT single crystals manufactured by Microfine Company (Singapore) [6].

TABLE I. PIEZOELECTRIC GENERATOR PARAMETERS

\begin{tabular}{|c|c|c|}
\hline \multirow{2}{*}{$\begin{array}{c}\text { Piezoelectric generator } \\
\text { characteristics }\end{array}$} & \multicolumn{2}{|c|}{ Piezoelectric generator parameters } \\
\cline { 2 - 3 } & Values & Units \\
\hline $\mathrm{D}$ & 0.16 & $\mathrm{~N} . \mathrm{s} . \mathrm{m}^{-1}$ \\
\hline $\mathrm{M}$ & 4.6 & $\mathrm{~g}$ \\
\hline $\mathrm{K}_{\mathrm{sc}}$ & 384.2 & $\mathrm{~N} . \mathrm{m}^{-1}$ \\
\hline$\alpha$ & 0.9 & $\mathrm{~mm} . \mathrm{s}^{-2}$ \\
\hline $\mathrm{C}_{\mathrm{p}}$ & 2.3 & $\mathrm{nF}$ \\
\hline $\mathrm{R}_{\mathrm{p}}$ & 10 & $\mathrm{M} \Omega$ \\
\hline $\mathrm{f}_{\mathrm{sc}}$ & 46 & $\mathrm{~Hz}$ \\
\hline $\mathrm{f}_{\mathrm{oc}}$ & 64 & $\mathrm{~Hz}$ \\
\hline $\mathbf{k}_{\mathbf{m}}^{2} \mathbf{Q}_{\mathbf{m}}$ & 7.3 & - \\
\hline
\end{tabular}




\section{B. Experimental results and discussion}

In the following part, we present and discuss the experimental results we obtained using the $N-S E C E$ strategy. We can observe the piezoelectric voltage for different $n$ on Fig. 7. The waveforms are similar to the theoretical ones (Fig. 3). We can clearly notice a piezoelectric voltage increase when $n$ gets greater. The harvested power experimentally is shown on Fig. 8. It confirms that the classic SECE overdamps the system, and hence is no longer optimal at the open circuit resonance frequency (here approximately $64 \mathrm{~Hz}$ ). For the piezoelectric generator we used, the maximum harvested power using the standard SECE $(n=1)$ is $2.43 \mu \mathrm{W}$. When $n$ equals 3 , this maximum becomes $4.1 \mu \mathrm{W}$, and $4.7 \mu \mathrm{W}$ when $n$ equals 5 . Hence, the optimal $n$ is 5 , leading to a $193 \%$ increase of the maximum harvested power. This $n$ is optimal on a frequency bandwidth of about $5 \mathrm{~Hz}$.

The differences between theory and practice are due to the dielectric losses (modeled by a resistance $R_{p}$ and neglected all along the theoretical analysis of this paper) that may have an impact on this strategy performance. Indeed, because of the regenerative behavior of the piezoelectric voltage, the dielectric losses are increased as $n$ gets greater, and are responsible for energy losses that are roughly proportional to the squared electric field in the material. This can explain the differences between the model and the experimental results around the piezoelectric generator open circuit resonant frequency, since this is where both the voltage and consequently the dielectric losses are the most important. These dielectric losses could be reduced with a different choice of piezoelectric material, and an optimized design of the electromechanical structure.

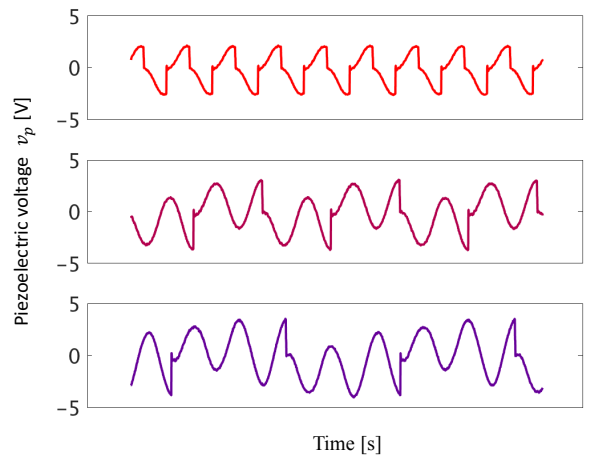

Fig. 7. Experimental waveforms of the piezoelectric voltage $v_{p}$ with $n=1,3,5$.

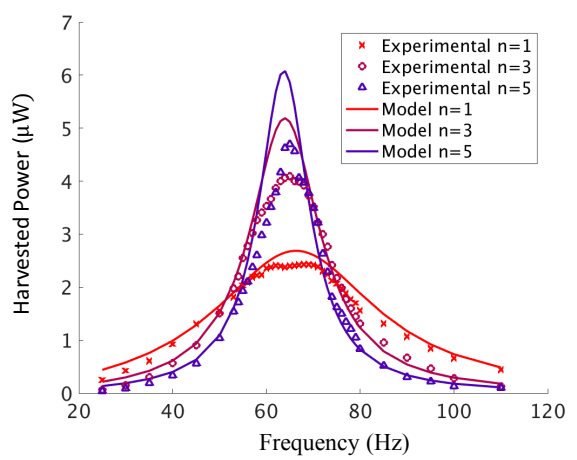

Fig. 8. Comparison between experimental harvested power and theoretical harvested power obtained with $n=1,3,5$ and $\ddot{y}=0.6 \mathrm{~m} . \mathrm{s}^{-2}$.

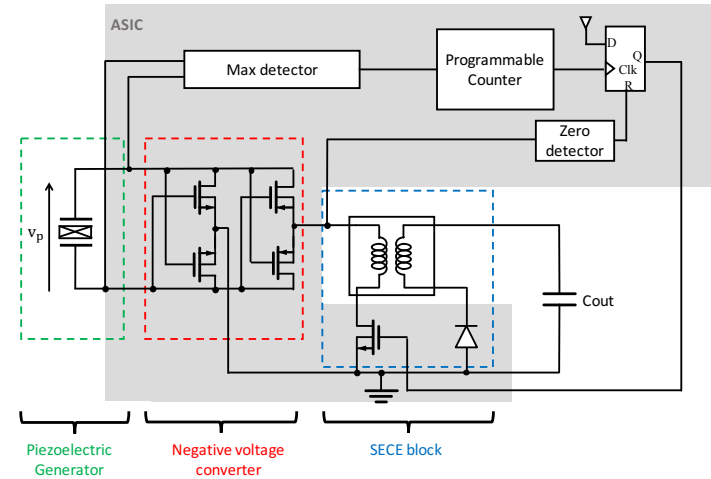

Fig. 9. Possible implementation of the N-SECE strategy.

\section{Implementation of the N-SECE strategy}

The implementation of the $N-S E C E$ relies on an extremum detection, just like the standard SECE. However, instead of directly connecting the inductance after a detection, a digital counter could be used in order to wait for the optimized number of extremum. The proposed implementation is shown on Fig. 9. The number of period between two harvesting events could be either fixed, or dynamically adjusted using a MPPT algorithm, depending on the application and on the kind of harvested vibrations.

\section{CONCLUSION}

In this paper, we propose a new harvesting strategy, the $\mathrm{N}$ $S E C E$. This strategy is a variant of the classic SECE: instead of harvesting the energy at every displacement extremum, it is harvested every $n$ extremum, in order not to overdamp the system. This strategy is particularly useful for highly coupled, lowly damped piezoelectric generators, when $k_{m}^{2} Q_{m}>2$, since it allows an electrical damping optimization.

Experimental results on a highly coupled piezoelectric generator composed of PZN-PT deposited on a cantilever have been presented. They demonstrate that the proposed strategy exhibits $193 \%$ improvement in extracted power at resonance.

Future work will focus on the integration of this strategy and on the implementation of a gradient algorithm that could tune dynamically the number of displacement extremum between every harvesting events.

\section{REFERENCES}

[1] E. Lefeuvre et al., "A comparison between several vibration-powered piezoelectric generators for standalone systems," Sensors and Actuators A: Physical, vol. 126, no. 2, pp. 405-416, Feb. 2006.

[2] P. Gasnier et al., "An Autonomous Piezoelectric Energy Harvesting IC Based on a Synchronous Multi-Shot Technique" IEEE Journal of SolidState Circuits, vol. 49, no. 7, pp. 1561-1570, Jul. 2014.

[3] T. Hehn et al., "A Fully Autonomous Integrated Interface Circuit for Piezoelectric Harvesters", IEEE Journal of Solid-State Circuits, vol. 47 no. 9, pp. 2185-2198, Sep. 2012.

[4] A. Morel et al., "Active AC/DC control for wideband piezoelectric energy harvesting," J. Phys.: Conf. Ser., vol. 773, no. 1, p. 12059, 2016.

[5] A. Richter et al., "Tunable interface for piezoelectric energy harvesting", in 2014 IEEE 11th International Multi-Conference on Systems, Signals Devices (SSD14), pp. 1-5, 2014.

[6] A. Badel et al., "Wideband Piezoelectric Energy Harvester Tuned Through its Electronic Interface Circuit," J. Phys.: Conf. Ser., vol. 557, no. 1, p. 12115,2014 